\title{
A COMPARATIVE STUDY OF WINDOWS FOR SPECTRAL ANALYSIS
}

\author{
D.V.L.N.Sastry, B. Anil Kumar, P.Kameswar Rao, V.Laxmi, A.Jayalaxmi, J.Swathi \\ Assistant Professors ECE Department \\ Aditya institute of technology and Management, Tekkali
}

\begin{abstract}
In this paper we presented a spectral analysis of different windows designed based on cosine functions. this paper includes comparative study of different windows based on their spectral characteristics like side lobe attenuation (SLA) half bandwidth (HBW) and side lobe fall of ratio (SLFOR).since windowing method one of the best method in the design of linear phase FIR filters using in digital signal processing applications like audio processing, video processing, speech processing and bio medical signal processing etc. Windows plays major role in design of Finite Length digital filters for removing pikes in stop band and improves pass band attenuation characteristics. And lot of research also done on windows. Many windows are developed based on their side lobe attenuation characteristics. Optimisation techniques also applied in the filed of window based FIR design, which shows better results compare to conventional methods.

Here we proposed basic mathematical kernel functions to develop window functions.
\end{abstract}

Key words: Bartlett window, Hamming window, Hanning window, proposed window functions.

\section{INTRODUCTION}

Windowing technique is used in to process the signals described by Fourier Transform modelled[1].FIR filters are designed by windowing method to remove the noises in ECG signals[2]. Windows are mathematical functions applied to both periodic signals and non periodic signals[3]. Windowing based FIR filters are used in communication systems to design of channel equalizers[4]. The magnitude and phase responses of FIR filters are important for selection of windows while designing.[5] the side lobe attenuation of spectral characteristics is key factor in the design of windows[6]. The sharper magnitude and liner phase response of FIR filters are obtained by windowing technique[7]. The stability and filter coefficients of FIR filters are varied for different window functions[8]. Window is a mathematical function allows the signal between the interval and zero value outside the interval[9]. Hybrid windows are designed which gives better results compare to basic window functions[10].

\section{WHY NEED WINDOWS IN SIGNAL PROCESSING}

When converting the continuous signals into discrete signals using sampling before transforming into frequency domain and transmitting there is a present of frequencies other than sampled instants referred as spectral leakage.

Windows are mathematical functions applied to signals either continuous or sampled to reduce the spectral leakage.

\section{BASIC WINDOW FUNCTIONS}

In this section we are presenting different presenting mathematical functions of windows used in the design of linear phase FIR filters.

\section{III.I Bartlett window}

The mathematical expression for Bartlett window is

$$
w(n)=\frac{2 n}{N}, 0 \leq n \leq \frac{N}{2}
$$

$=2-\frac{2 n}{N}, \frac{N}{2} \leq n \leq N$

\section{III.II Hamming window}

The mathematical expression for Hamming window is

$$
\begin{aligned}
& w(n)=0.54-0.46 \cos \left(\frac{2 \pi n}{N}\right), \quad 0 \leq n \leq N-1 \\
& =0 \text { otherwisw }-
\end{aligned}
$$

\section{III.III Hanning window}

The mathematical expression for Hanning window is

$$
\begin{aligned}
& w(n)=0.5-0.5 \cos \left(\frac{2 \pi n}{N}\right), \quad 0 \leq n \leq N-1 \\
& \quad=0 \text { otherwise }
\end{aligned}
$$

\section{PROPOSED WINDOWS}

The window functions are generally continuous cosine functions implemented as a powers of integers of cosine functions which are referred as cosine windows given as

$$
\begin{aligned}
w(n) & =\cos ^{\propto}\left[\frac{n \pi}{N}\right], \quad \frac{-N}{2} \leq n \frac{N}{2} \\
& =0 \quad \text { otherwise ----- }
\end{aligned}
$$


But it is also confirmed that windows are not only continuous but also developed from their second derivatives called as Dirchlet Kernels. The Dirchlet kernels are located about the central kernel which are zeros of central kernel. So the summation of kernels side lobes results the cancellation of side lobes because of opposite phases. so this partial structure of side lobes with three or more kernel functions results to construction of new windows which are described in mathematical function is

$$
\begin{aligned}
w(n)=x_{0} \cos \frac{2 \pi n}{N}-x_{1} \cos \frac{4 \pi n}{N} \\
\\
\quad+x_{2} \cos \frac{4 \pi n}{N}-x_{3} \cos \frac{6 \pi n}{N}+\cdots . .
\end{aligned}
$$

Where $\mathrm{x}=0,1,2,3----\mathrm{N}-1$

The coefficients $\mathrm{x}$ determines $\mathrm{n}$-tem window where $\mathrm{n}$ is the number of coefficients. So here we proposed the windows with 3 terms and 4 terms and observed their spectral responses with basic conventional windows.

IV.I Proposed window1: It is a 3 term window and the mathematical function is

$$
\begin{aligned}
w(n)=0.42323 & \cos \frac{2 \pi n}{N} \\
& -0.49755 \cos \frac{4 \pi n}{N}+0.07922 \cos \frac{6 \pi n}{N}
\end{aligned}
$$

IV.I Proposed window2: It is a 4 term window and the mathematical function is

$$
\begin{aligned}
w(n)=0.35875 & \cos \frac{2 \pi n}{N} \\
& -0.48829 \cos \frac{4 \pi n}{N} \\
& +0.14128 \cos \frac{6 \pi n}{N}-0.01168 \cos \frac{8 \pi n}{N} \\
\text { V. } & \text { SIMULATION \& RESULTS }
\end{aligned}
$$

In this sections we developed simulation results for spectral responses of Bartlett window, Hamming window, Hanning window and two proposed windows. The simulation results are obtained from MATLAB.

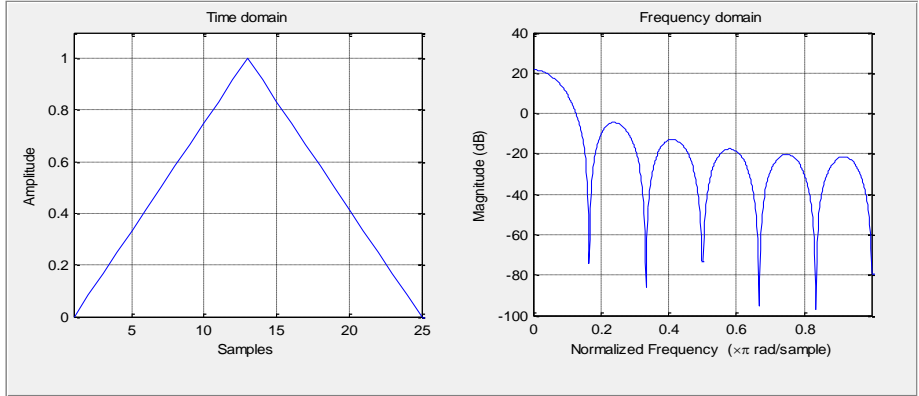

Fig1: Spectral response of Bartlett window

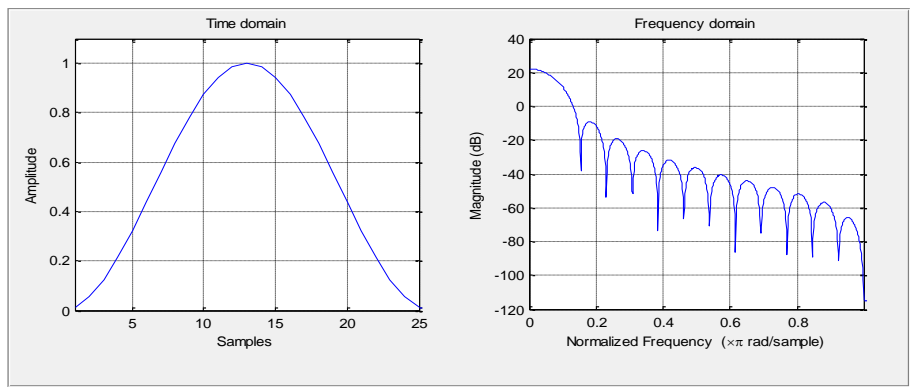

Fig2: Spectral response of Hanning window
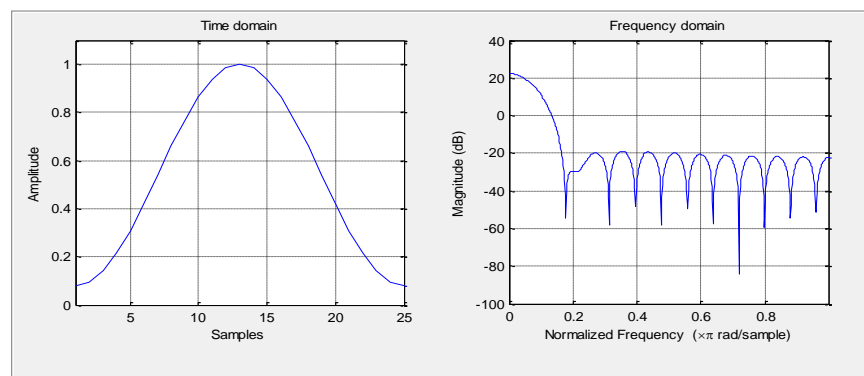

Fig3: Spectral response of Hamming window

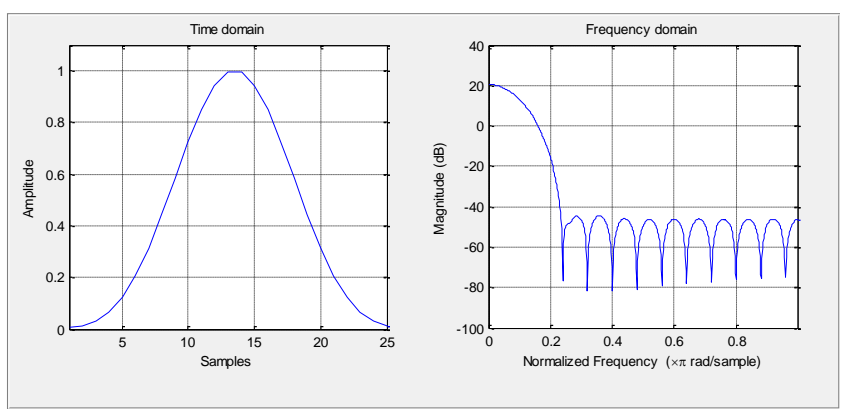

Fig4: Spectral response of proposed window1 

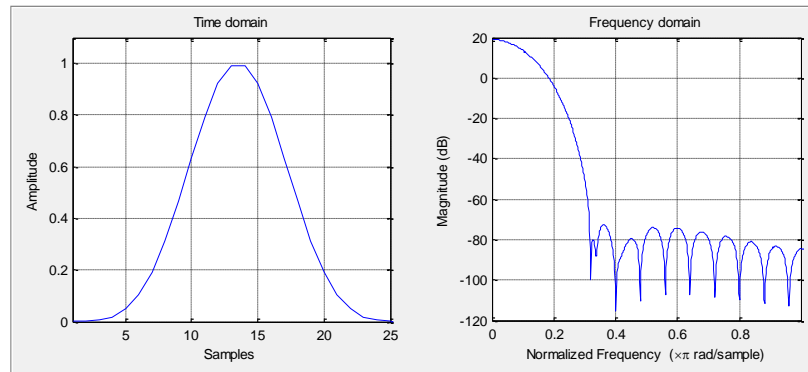

Fig5: Spectral response of proposed window2

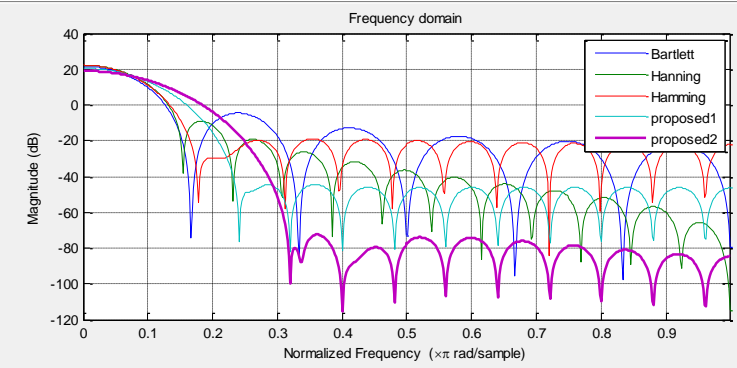

Fig6: Spectral response of all windows

Table1: Spectral characteristics of windows

\begin{tabular}{|c|c|c|c|c|}
\hline S.no & Window & $\begin{array}{c}\text { Side lobe } \\
\text { attenuation } \\
\text { SLA(dB) }\end{array}$ & $\begin{array}{c}\text { Half bandwidth } \\
\text { HBW(db) }\end{array}$ & $\begin{array}{c}\text { Side } \\
\text { lobe fall } \\
\text { of ratio }\end{array}$ \\
\hline 1 & Bartlett & -26.1 & 0.10156 & 17.28 \\
\hline 2 & Hanning & -31.5 & 0.10938 & 56.36 \\
\hline 3 & Hamming & -41.2 & 0.10156 & 7.39 \\
\hline 4 & Proposed1 & -65 & 0.125 & 1.45 \\
\hline 5 & Proposed2 & -91.6 & 0.14844 & 12.03 \\
\hline
\end{tabular}

\section{CONCLUSION}

We have examined some cosine function based windows and proposed windows which satisfies their performance towards spectral leakage and harmonic analysis. for multiple speech detection through DFT the windows should exhibit concentrated central lobe width and narrow side lobes which results sharp attenuation in stop band of the filter. We have demonstrated the proposed windows which exhibits higher side lobe attenuation i.e $-65 \mathrm{~dB}$ for 3 term window (proposed window1) and $-91.6 \mathrm{~dB}$ for 4 term window (proposed window2). See Table1 for reference. And we also presented spectral time and frequency domain analysis of all windows shown from Fig1 to Fig6.

\section{REFERENCES}

[1] Fredric J.harris, 'On the use of windows for Harmonic Analysis with the discrete Fourier Transform", Proceedings of the IEE vol:66, no:1,1978

[2] Dvln Sastry,K.Srinadh gupta, V.mohan krushana, DSVS Nagendra reddy, "IMPROVED SNR OF ECG SIGNAL WITH NEW WINDOW- FIR DIGITAL FILTERS ", International Journal of Advanced Research in Electronics and Communication Engineering (IJARECE) Volume 1, Issue 3, September 2012

[3] Mitun Shil, Hrishi Rakshit, Hadaate Ullah," An Adjustable Window Function To Design An FIR Filter', 978-1-5090-6004-7/@2017IEEE

[4] Manira Khatun," IMPLEMENT A NEW WINDOW FUNCTION AND DESIGN FIR FILTERS BY USING THIS NEW WINDOW', International Journal Of Engineering And Computer Science ISSN:2319-7242 Volume- 3, Issue -3 , March, 2014 Page No. 4087-4090

[5] Suhaib Ahmed,' Design Analysis of High Pass FIR Filters Using Hanning, Bartlett and Kaiser Windows", International Journal of Advanced Research in Computer Science and Software Engineering, Volume 2, Issue 11, November 2012 ISSN: 2277 128X

[6] Suhaib Ahmed, Mudasir Bashir, Ashish Suri," "Low Pass FIR Filter Design and Analysis Using Hamming, Blackman and Kaiser Windows", International Journal of Advanced Research in Electrical, Electronics and Instrumentation Engineering (An ISO 3297: 2007 Certified Organization) Vol. 3, Issue 4, April 2014

[7] Girdhar chandra, Hemlal sahu, Pranay Kumar Rahi," DESIGN OF LOWPASS FIR FILTER USING NUTTALL, TAYLOR AND TUKEY WINDOW TECHNIQUES", International Journal of Scientific Research Engineering \& Technology (IJSRET), ISSN 2278 - 0882 Volume 6, Issue 6, June 2017

[8] Sonika Aggarwal, Aashish Gagneja, Aman Panghal "International Journal of Advanced Research in Computer Science and Software Engineering", Volume 2, Issue 7, July 2012 ISSN: 2277 128X

[9] Qeethara Al-Shayea , Muzhir Al-Ani,''Efficient Window Approach of FIR Filter Design", IJCSNS International Journal of Computer Science and Network Security, VOL.16 No.2,2016 [10]EPPILI JAYA, K.CHITAMBARA RAO, JAYA LAXMI. ANEM," FIR FILTER DESIGN USING NEW HYBRID WINDOW FUNCTIONS", International Journal of Science, Engineering and Technology Research (IJSETR), Volume 4, Issue 6, June 2015 